\title{
Effectiveness of Yoga Lifestyle on Lipid Metabolism in a Vulnerable Population-A Community Based Multicenter Randomized Controlled Trial
}

\author{
Raghuram Nagarathna ${ }^{1, *,+} \ddagger$, Saurabh Kumar ${ }^{2,+} \mathbb{D}$, Akshay Anand $2,3,4, *, \ddagger$, Ishwara N. Acharya ${ }^{5}$, \\ Amit Kumar Singh ${ }^{1}$, Suchitra S. Patil ${ }^{1}$, Ramesh H Latha ${ }^{6}$, Purnima Datey ${ }^{7}$ and Hongasandra Ramarao Nagendra ${ }^{1}$ \\ 1 Swami Vivekananda Yoga Anusandhana Samsthana (S-VYASA), Bengaluru 560105, India; \\ dramits90@gmail.com (A.K.S.); ayursuch@gmail.com (S.S.P.); chancellor@svyasa.edu.in (H.R.N.) \\ 2 Neuroscience Research Lab, Department of Neurology, Postgraduate Institute of Medical Education and \\ Research (PGIMER), Chandigarh 160012, India; Saurabh.kum1991@gmail.com \\ 3 Centre for Mind Body Medicine, PGIMER, Chandigarh 160012, India \\ 4 Centre for Cognitive Science and Phenomenology, Panjab University, Chandigarh 160014, India \\ 5 Central Council for Research in Yoga \& Naturopathy (CCRYN), Delhi 110058, India; \\ acharyaishwar@gmail.com \\ 6 Yoga Clinic, Bhopal 462026, India; latavk123@gmail.com \\ 7 Arogya Rasahara Kendra, Bhopal 462024, India; purnimadatey@gmail.com \\ * Correspondence: rnagaratna@gmail.com (R.N.); akshay1anand@rediffmail.com (A.A.) \\ + Equal first authors. \\ ‡ Corresponding author: Raghuram Nagarathna; Co-corresponding author: Akshay Anand.
}

Citation: Nagarathna, R.; Kumar, S.; Anand, A.; Acharya, I.N.; Singh, A.K.; Patil, S.S.; Latha, R.H; Datey, P.; Nagendra, H.R. Effectiveness of Yoga Lifestyle on Lipid Metabolism in a

Vulnerable Population-A

Community Based Multicenter Randomized Controlled Trial. Medicines 2021, 8, 37. https:// doi.org/10.3390/medicines 8070037

Academic Editors: Roberto Anichini and Piergiorgio Francia

Received: 1 April 2021

Accepted: 29 June 2021

Published: 13 July 2021

Publisher's Note: MDPI stays neutral with regard to jurisdictional claims in published maps and institutional affiliations.

Copyright: () 2021 by the authors. Licensee MDPI, Basel, Switzerland. This article is an open access article distributed under the terms and conditions of the Creative Commons Attribution (CC BY) license (https:// creativecommons.org/licenses/by/ $4.0 /)$.
Abstract: Background: Dyslipidemia poses a high risk for cardiovascular disease and stroke in Type 2 diabetes (T2DM). There are no studies on the impact of a validated integrated yoga lifestyle protocol on lipid profiles in a high-risk diabetes population. Methods: Here, we report the results of lipid profile values of 11,254 (yoga 5932 and control 5322) adults (20-70 years) of both genders with high risk ( $\geq 60$ on Indian diabetes risk score) for diabetes from a nationwide rural and urban community-based two group (yoga and conventional management) cluster randomized controlled trial. The yoga group practiced a validated integrated yoga lifestyle protocol (DYP) in nine day camps followed by daily one-hour practice. Biochemical profiling included glycated hemoglobin and lipid profiles before and after three months. Results: There was a significant difference between groups $(p<0.001$ ANCOVA) with improved serum total cholesterol, triglycerides, low-density lipoprotein, and high-density lipoprotein in the yoga group compared to the control group. Further, the regulatory effect of yoga was noted with a significant decrease or increase in those with high or low values of lipids, respectively, with marginal or no change in those within the normal range. Conclusion: Yoga lifestyle improves and regulates (lowered if high, increased if low) the blood lipid levels in both genders of prediabetic and diabetic individuals in both rural and urban Indian communities.

Keywords: diabetes yoga protocol; diabetes; prediabetes; dyslipidemia; lipid regulation

\section{Introduction}

Dyslipidemia (altered blood lipids) is as a contributing risk factor for various macrovascular complications in type-2 diabetes mellitus (T2DM) patients [1]. Dyslipidemia is characterized by high levels of triglycerides ( $\geq 150 \mathrm{mg} / \mathrm{dL}$ ), high low-density lipoprotein (LDL $\geq 130 \mathrm{mg} / \mathrm{dL}$ ), low high-density lipoprotein (HDL $<40 \mathrm{mg} / \mathrm{dL}$ for men; $<50 \mathrm{mg} / \mathrm{dL}$ for women) [2] and high levels of total cholesterol ( $\geq 200 \mathrm{mg} / \mathrm{dL})$ [3,4]. It is difficult to define the cut-off range for dyslipidemia, as it varies from study to study due to the difference in methodologies used. Studies suggest that Indian and migrant Asian Indians tend to show increased triglycerides and decreased HDL serum levels than western residents [5]. In comparison, serum cholesterol levels tend to be similar to the US population 
and lower than the UK population [5]. A high volume of blood cholesterol is associated with greater chances of developing cardiovascular disease, including stroke, peripheral vascular disease, and coronary heart disease (CHD) [6]. This is a major cause of cardiac morbidity and mortality, especially in the aged and in patients with T2DM [6]. According to the American Diabetes Association (ADA), T2DM is associated with a two- to four-fold increased risk of developing CHD and increased triglycerides. Decreased HDL levels are common in patients with T2DM [7,8]. The Indian Council of Medical Research study revealed a high prevalence of dyslipidemia in India, with $79 \%$ of the studied population showing an abnormality in at least one lipid parameter [9]. The study found a higher prevalence of abnormal lipids in females than in males. The middle-aged group population (35-64 yrs) showed higher lipid abnormalities than the younger group (20-24 yrs) [9].

The increasing burden of heart disease and T2DM [10], despite billions of dollars spent on research and the use of lipid-lowering drugs over the years, has posed a big challenge for health expenditure [11], and there is an urgent need to investigate cost-effective alternative approaches. Yoga is one of the popular mind-body approaches developed in India [12]. Yoga is known to exert positive physiological changes, which have wide-ranging scientific significance $[13,14]$ as research findings have described the benefits of yoga in managing stress, anxiety, and negative sentiments [12,15]. Yoga may exert cardiovascular changes by acting on neurological pathways like the autonomic nervous system (ANS), sympathoadrenal medullary (SAM), or hypothalamic pituitary adrenal (HPA) [16]. It is also believed that yoga postures like pranayama (breathing) and asanas improve cardiovascular and respiratory activity by increasing nitric oxide $(\mathrm{NO})$ and antioxidant levels in the blood. Further, the HPA/SAM are hypothesized to reduce the over-production or activation of catecholamines, corticosteroids (glucocorticoids), and subsequent cytokines that are pro-inflammatory, increasing CHD risk $[16,17]$. $\beta$-cell sensitivity in response to glucose metabolism and insulin secretion is improved by these yoga postures [18].

Hypercholesterolemia, hypertriglyceridemia, and hyperlipidemia are significant risk contributors for coronary heart disease. Both prevention and control of coronary heart disease with its associated diseases are essential and can be achieved by modifying the lipid profile [19]. There are many reports on the adverse effects of an increased volume of bad cholesterol (LDL, triglycerides) and reduced volume of good cholesterol (HDL) and on drugs to reduce LDL and increase HDL levels. Some studies have shown the detrimental effects (increased mortality in coronary heart disease) of lowering the levels of cholesterol ( $<160 \mathrm{mg} / \mathrm{dL})$, which calls for the integration of an effective evidence-based non-pharmacological approach (Cir and EHJ) [20,21]. Mahajan et al. conducted a yogabased study on the lipid profiles of subjects with coronary artery disease and reported the effectiveness of yoga in risk modification [22]. Another study reported yoga exerted its therapeutic potential in subjects with mild-to-moderate hypertension by reducing the risk of cardiovascular diseases [23]. A systemic review conducted by Innes et al. evaluated the effects of yoga-based controlled trials and found yoga to be effective in managing the blood lipids along with glycemic control [24]. Similarly, Raveendran et al. [25] suggested that daily yoga practice helps maintain overall body growth [25]. Hence, it appears that maintaining the lipid levels within the normal range is essential [26], which can be achieved by integrating yoga with usual care.

There are a few studies on lipid profiles in T2DM patients [27,28], but there are none that have examined the normalizing effect of yoga on lipid values. This requires a large sample size from diverse community cohorts, which is challenging. Hence, the present paper planned to look at the lipid normalizing effect of the yoga lifestyle change program in a trial that was designed as a nationwide multicenter two-armed control trial for primary and secondary prevention of diabetes named Niyantrita Madhumeha Bharata Abhiyaan (NMB2017). The sampled population is vulnerable (high risk) to diabetes with scores $\geq 60$ on the Indian Diabetes Risk Score (IDRS). 


\section{Materials and Methods}

\subsection{Sample Size Calculation}

The sample size for the trial was calculated for the primary prevention of diabetes, which was the primary objective of the study. The details of the sample size calculation are provided in our earlier publication [29]. In brief, we used the values of relative riskreduction after lifestyle intervention in prediabetes subjects, as observed in an earlier study [30], which had an annual conversion rate of $11.1 \%$ in the control and $7.8 \%$ in the intervention group; based on this we obtained a total sample size of 5320, i.e., 2660/group at $\alpha=0.05$ and $1-\beta=0.80$.

\subsection{Screening and Recruitment}

The screening was carried out after getting permission from the Institutional Ethics Committee of the Indian Yoga Association (IYA) (Reference no: RES/IEC-IYA/001) and obtaining the signed informed consent by all participants. Details of the methodology are communicated in an earlier publication [31]. This was multi-level randomization starting from the randomization of districts, towns, and census enumeration blocks (urban) and villages (rural) depicted in the map (Figures 1 and S1) [31]. The study was a cluster randomization design in order to overcome the barriers of contamination. For this reason, we included the entire block or village for yoga intervention or control as the case may be. The yoga and control clusters were separated by 5-10 km. We randomly identified the group as an intervention group, i.e., 2 out of 4 villages and 1 or 2 out of 2 or 4 census enumeration blocks (CEBs) were identified in the selected ward, while the other was assigned as the waitlisted control group. Phase 2 included administering the yoga lifestyle protocol for diabetes developed by an expert committee through the Delphi method in the randomly allocated clusters.

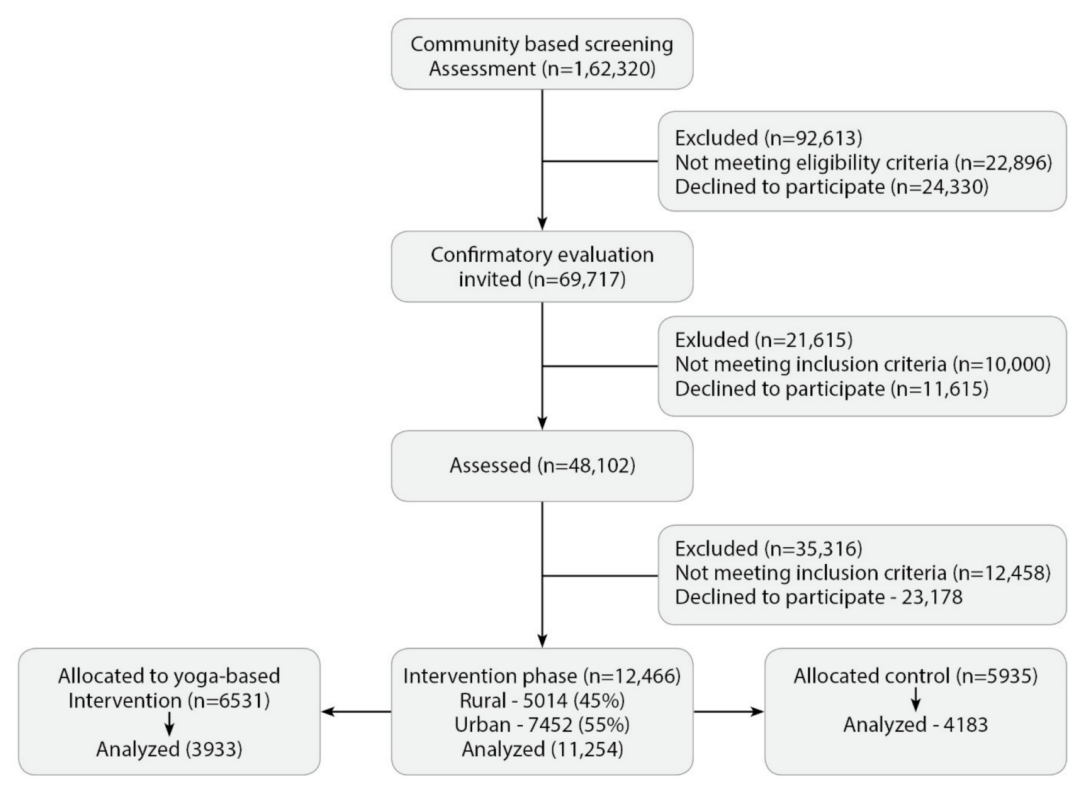

Figure 1. CONSORT diagram of the study.

\subsection{Randomization and Allocation Concealment}

Details of randomization have been published in our earlier publication [29]. In brief, a 4-stage randomization approach was implemented using a multi-level stratified cluster sampling method. The study was planned to be in two phases. Phase 1 involved a cross-sectional survey from the entire country using the National Family Health-3 (NFH-3) sampling process. The twenty-nine (95\% of India's population from 2011 census) most populous states/union territories of India were grouped under seven geographical zones (Northwest, North, Northeast, West, Central, East, and South) based on their cultural 
similarities (Figure S1); random selection process was applied for selecting 65 districts, four rural villages and two census enumeration blocks in an urban town. In brief, this was a trial on randomly selected rural and urban community clusters. Phase 1 was door-to-door screening for IDRS in these randomly selected clusters of rural (villages) and urban (CEBs) locations, followed by blood tests in high-risk ( $\geq 60$ on IDRS) individuals.

\subsection{Selection Criteria for Phase 2 (RCT)}

In phase 2, two villages and one CEB were randomly selected for yoga intervention, and two villages and one CEB formed waitlist control. Adults (20 years) of both genders with IDRS Score $\geq 60$ and those with known diabetes (any score on IDRS) were included. Those with reported psychiatric problems, major diabetes complications (nephropathy, retinopathy, coronary artery disease, history of cerebrovascular accidents) were excluded. Pregnant women, lactating mothers, and those who had any surgery within 12 months were also excluded. Information about medication was taken. Individuals taking drugs were excluded from the study. The lipid values of those who had reported that they had practiced yoga regularly within the last three months before the camp were not included in this analysis.

\subsection{Blinding and Masking}

Since it was a community-based interventional cluster-randomized trial, the participants, instructors, and other individuals involved in the study were not blinded. Masking was ensured at different levels. The researchers in the central office provided the names of the randomly selected names of clusters to the field Senior Research Fellows (SRFs), and hence the selection bias was avoided. The central laboratory that carried out the blood investigations on the coded samples, the data operator who checked the accuracy of the data obtained online from the field researchers, and the statistician who analyzed the data were blinded.

\subsection{Assessments}

Zonal coordinators, yoga volunteers, and SRFs visited the campsites for formal interaction with the leaders of the towns/villages and conducted door-to-door screening using the IDRS parameters. Those eligible based on the inclusion/exclusion criteria were invited to the blood camp. Two phlebotomists drew the blood samples in coded label vacutainers. The sample was processed for plasma separation, stored and transported in cold containers to the nearest laboratory, and was processed for biochemical analysis within $6 \mathrm{~h}$ of blood withdrawal. Data was collected by SRL labs. For storing, the sample standard method was applied. These estimations were carried out by the same NABL accredited laboratory (SRL) on fasting blood samples collected on day one and after three months of the trial in both yoga and control groups. Serum from the fasting venous blood sample was used for the estimation of lipids and glycated hemoglobin (A1c) on an auto-analyzer (Beckman CoulterAuto-analyzer model 2700/480); the cholesterol esterase oxidase-peroxidase-amidopyrine method for TC, the glycerol phosphate oxidase-peroxidase-amidopyrine method for TG and polyethylene glycol-pretreated enzymes for HDL [29,31]. The criteria for diabetes and prediabetes were based on A1c values. ADA guidelines recommend A1c based screening as the most practical measure to segregate the population into healthy (A1c $<5.3 \%$ ), prediabetes (A1c: $5.3-6.4 \%$ ), and diabetes (A1c $\geq 6.5 \%$ ) considering the impending practical challenges in screening a large population.

\subsection{Quality Assurance and Training}

Each zone had a zonal coordinator, 35 SRFs (approximately 1 per 2 districts), 1200 certified yoga volunteers, and 2 research associates. The zonal training program organized in different zones trained the SRFs in organizing the camps, data acquisition, volunteer training, using the mobile app, maintaining logbooks, conducting regular meetings, etc. 
These SRFs further trained the volunteers in their respective areas. We used both paper and mobile apps to capture the data, collated by deep learning and big data analysis.

\subsection{Intervention}

Both groups were given the standard medical advice on lifestyle for the prevention and management of diabetes under the doctor's supervision from the local medical center. The advice on lifestyle through detailed interactive group lectures included (a) advice on a healthy diet for diabetes; (b) regular and timely exercise (walking for more than 20 min daily); (c) habits (sleep, hygiene, tobacco, alcohol, mobile addiction etc.); and (d) stress management.

\section{Diabetes Yoga Protocol}

The yoga group received a validated diabetes yoga lifestyle protocol (DYP) developed by an expert committee of 16 professionals (yoga masters, yoga researchers, and diabetologist) with two rounds of interaction using the Delphi technique with a CVR $>0.7$. The reliability of the protocol was tested by cluster analysis with an interclass coefficient value of 0.05. The details of the expert committee are published earlier [29].

The 60-min yoga protocol (Table S1) for prediabetes and uncomplicated diabetes consisted of yoga postures, breathing practices, relaxation, pranayama, meditation, and lectures on yogic lifestyle for behavioral modification (diet, sleep, stress management through conceptual correction using jnana yoga, bhakti yoga and karma yoga). During the initial 9-day introductory camps, daily feedback for any adverse effect was recorded. After this period, they were asked to do the practices at home using handbooks and/or DVDs; adverse effects were discussed and documented during the weekly follow-up classes of $2 \mathrm{~h}$ for three months. Fidelity data was documented through the attendance sheet and regular follow-up calls on the phone; individual pictures and videos were also recorded and archived in a retrievable format. No financial incentive was given to the participants. Any adaptation was decided locally by the trained instructors, which was done based on individual cases and not for the area as a whole. The standard protocol was implemented in all places uniformly by trained, certified instructors, e.g., some patients who could not squat on the floor for physical postures were taught a modified version of the postures to be done sitting in a chair. This was included and taught to the instructors in their 5-day orientation programs ( 5 days each in 20 orientation camps in different zones) [31].

\subsection{Statistical Analysis}

Data were analyzed using SPSS version 21.0. The matching of data from different sources and different time points was checked by fuzzy logic. Independent samples $t$-test was used to compare baseline characteristics of the two groups. Paired samples $t$-test was used for pre-post comparison within groups. Pre-post comparisons between yoga and control groups were checked by the ANCOVA test. The difference in deference analysis was done by multinomial regression. A mixed linear model was used to check the differences between subcategories of lipid values in 3 subgroups of A1c.

\section{Results}

Figure 1 shows the consort diagram. In the first phase of pan-India screening from seven zones (Figure S1), data on IDRS and known diabetes were available on 162,330 individuals from the randomized urban (52\%) and rural (48\%) clusters. Of these, 69,717 individuals at high risk and who had known diabetes were invited for detailed assessments, and 48,102 responded. Based on A1c values, 6094 were found to be newly diabetic (A1c $\geq 6.5$ ), 7920 were in the prediabetes range, and 13,597 were in the normoglycemia range and were invited for intervention in phase 2 of the trial. Of the 12,466 who participated in the trial, 6531 were in the yoga clusters and 5935 in the control clusters from all zones. The main reason for non-response, although they were interested in participation, was time constraints due to family or occupational commitments. Of these, follow-up data were 
available on 11,254 (5932 yoga and 5322 control; 9\% drop out) at three months; analysis of pre-post lipid profile data was done on 8116 (3933 yoga and 4183 control) individuals after excluding extreme values.

The baseline characteristics (Table 1) revealed a non-significant difference between groups in the age and gender distribution (independent samples $t$-test) between the yoga and control groups. The mean (SD) age (years) of the yoga and control groups was $48.70 \pm 10.64$ of $48.41 \pm 10.22$, respectively. In the yoga group, the percentage of the male population was $42.8 \%$, and in the control group, it was $40.9 \%$. In the yoga group, the proportion of the rural population was $31.5 \%$, while in the control, it was $47.3 \%$. There were 4896 subjects in urban (2693 yoga and 2203 control) and 3220 subjects in rural locations (1240 yoga and 1980 control). By profession, most of the recruited participants were clerical or shop owners, followed by skilled workers. A total of 502 (n) participants in the yoga group and 616 (n) in the control group were aware of their diabetes status/that they had diabetes for the last five years. Many participants were newly diagnosed with diabetes (1105 in the yoga group and 2145 in the control group) (Table 1).

Table 1. Demographic details of yoga and control groups.

\begin{tabular}{|c|c|c|c|c|c|}
\hline \multicolumn{3}{|c|}{ Demographic Details } & Yoga & Control & $p$ Value \\
\hline Age (years) & \multicolumn{2}{|l|}{ Mean \pm SD } & $48.7 \pm 10.64$ & $48.41 \pm 10.22$ & 0.03 \\
\hline \multirow{2}{*}{ Gender } & \multicolumn{2}{|l|}{ Male N (\%) } & $1682(42.8 \%)$ & $1710(40.9 \%)$ & \multirow{2}{*}{$<0.001$} \\
\hline & \multicolumn{2}{|l|}{ Female N (\%) } & $2251(57.2 \%)$ & $2473(59.1 \%)$ & \\
\hline \multirow{2}{*}{ Area } & \multicolumn{2}{|l|}{ Urban N (\%) } & $2693(69.5 \%)$ & $2203(52.7 \%)$ & \multirow{2}{*}{$<0.001$} \\
\hline & \multicolumn{2}{|l|}{ Rural N (\%) } & $1240(31.5 \%)$ & $1980(47.3 \%)$ & \\
\hline \multirow{6}{*}{ Occupation } & \multicolumn{2}{|l|}{ Profession } & 594 & 778 & \multirow{6}{*}{0.05} \\
\hline & \multicolumn{2}{|l|}{ Semi-Profession } & 106 & 130 & \\
\hline & \multicolumn{2}{|l|}{ Clerical, Shop owner } & 1415 & 1405 & \\
\hline & \multicolumn{2}{|l|}{ Skilled worker } & 1321 & 1345 & \\
\hline & \multicolumn{2}{|l|}{ Semi-skilled worker } & 90 & 95 & \\
\hline & \multicolumn{2}{|l|}{ Unskilled worker } & 153 & 170 & \\
\hline \multirow{6}{*}{ Diabetes status } & \multirow{3}{*}{$\begin{array}{l}\text { Self-declared } \\
\text { Known DM }\end{array}$} & $<5$ yrs & 502 & 616 & \multirow{6}{*}{$<0.001$} \\
\hline & & $5-10$ yrs & 163 & 220 & \\
\hline & & $>10$ yrs & 160 & 209 & \\
\hline & \multicolumn{2}{|l|}{ Newly diagnosed DM } & 1105 & 2145 & \\
\hline & \multicolumn{2}{|l|}{ Pre-diabetes } & 806 & 678 & \\
\hline & \multicolumn{2}{|c|}{ No DM, only high risk on IDRS } & 1197 & 315 & \\
\hline
\end{tabular}

The group analysis (Table 2) showed that TC was reduced significantly $(p<0.001$ paired t-test) from $181.80 \pm 39.75 \mathrm{mg} / \mathrm{dL}$ to $176.64 \pm 38.59 \mathrm{mg} / \mathrm{dL}$ after yoga, while there was an increase in the control group from $183.44 \pm 40.33 \mathrm{mg} / \mathrm{dL}$ to $193.27 \pm 47.27 \mathrm{mg} / \mathrm{dL}$. A marginal increase (153.51 $\pm 72.88 \mathrm{mg} / \mathrm{dL})$ in TG was observed in the yoga group after three months, whereas in the control group, the mean value increased from $155.86 \pm 79.40 \mathrm{mg} / \mathrm{dL}$ to $191.12 \pm 107.44 \mathrm{mg} / \mathrm{dL}$. We found a significant reduction in LDL in the yoga group from $103.54 \pm 34.09 \mathrm{mg} / \mathrm{dL}$ to $98.65 \pm 33.67 \mathrm{mg} / \mathrm{dL}$; the control group instead showed an increase in LDL from $103.99 \pm 33.00 \mathrm{mg} / \mathrm{dL}$ to $108.01 \pm 40.4 \mathrm{mg} / \mathrm{dL}$. Blood HDL showed a reduction in both groups; the yoga group reduced marginally from $49.30 \pm 11.48 \mathrm{mg} / \mathrm{dL}$ to $48.61 \pm 11.55 \mathrm{mg} / \mathrm{dL}$; similarly, the control decreased from $48.92 \pm 11.53 \mathrm{mg} / \mathrm{dL}$ to $44.62 \pm 12.15 \mathrm{mg} / \mathrm{dL}$. Similar changes were found in TG, LDL, and HDL (Table 2).

Analysis of covariance (Table 2) between groups showed that there was a significantly better improvement in the yoga than the control group in TC, TG, LDL, and HDL $(p<0.001$ 
ANCOVA). There was a significant difference between groups ( $p<0.001$ Mixed Linear Model analysis) in two subgroups (Table 3) of A1c, i.e., in individuals in the diabetic and prediabetic ranges. There was a non-significant difference between the yoga and control groups in the normoglycemic subgroup.

An interesting observation emerged in the yoga group when we looked at the three subcategories of baseline lipid values, i.e., those with less than, more than, or within the normal range (Table 3, Figure S2). In diabetics (A1c $\geq 6.5$ ) with baseline levels of TC above the normal range, there was a significant reduction, and for those below the normal range, there was a significant increase with a non-significant change in those within the normal range. Looking at TG, LDL, and HDL, there was a significant reduction in those within and above normal ranges with an increase in those below the normal range. This phenomenon of a shift towards normalcy was not seen in the control group. Although there was a significant increase in those below normal values, there was a significant increase in those with average and high TC, TG, LDL, and HDL values. Sub-group analyses showed that there were no significant differences between males and females, urban and rural areas, or young ( $<40 \mathrm{yrs})$ and old ( $>40 \mathrm{yrs})$ age groups $(p>0.05$ ANCOVA) in any of the lipid variables (Table 4). 
Table 2. Changes in lipid variables before and after three months in the two groups. $N=8116$ (Yoga-3933, Control-4183).

\begin{tabular}{|c|c|c|c|c|c|c|c|c|}
\hline Group & TC_Pre $(\mathrm{mg} / \mathrm{dL})$ & TC_Post (mg/dL) & TG_Pre (mg/dL) & TG_Post (mg/dL) & LDL_Pre (mg/dL) & $\begin{array}{c}\text { LDL_Post } \\
\text { (mg/dL) }\end{array}$ & HDL_Pre $(\mathrm{mg} / \mathrm{dL})$ & $\begin{array}{c}\text { HDL_Post } \\
\text { (mg/dL) }\end{array}$ \\
\hline Yoga & $181.80 \pm 39.75$ & $176.64 \pm 38.59 *+$ & $150.42 \pm 70.52$ & $153.51 \pm 72.88^{*}+$ & $103.54 \pm 34.09$ & $98.65 \pm 33.67^{*} \dagger$ & $49.30 \pm 11.48$ & $48.61 \pm 11.55^{*}+$ \\
\hline Control & $183.44 \pm 40.33$ & $193.27 \pm 47.27$ * & $155.86 \pm 79.40$ & $191.12 \pm 107.44$ * & $103.99 \pm 33.00$ & $108.01 \pm 40.4^{*}$ & $48.92 \pm 11.53$ & $44.62 \pm 12.15^{*}$ \\
\hline
\end{tabular}

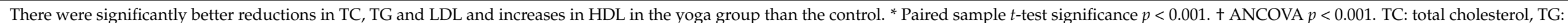

triglycerides, LDL: low-density lipoprotein, HDL: high-density lipoprotein.

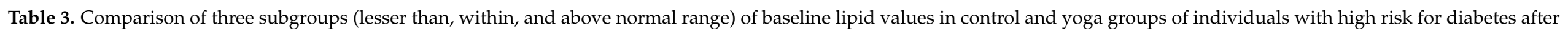
three months of intervention. TC: Total Cholesterol; TG: Triglyceride; LDL: low-density lipoprotein; HDL: high-density lipoprotein.

\begin{tabular}{|c|c|c|c|c|c|c|c|c|c|c|}
\hline \multirow[t]{2}{*}{ Groups } & \multirow{2}{*}{$\begin{array}{c}\text { Lipid } \\
\text { Categories }\end{array}$} & \multicolumn{3}{|c|}{ Diabetes A1c $(\geq 6.5)$} & \multicolumn{3}{|c|}{$\begin{array}{c}\text { Prediabetes (A1c 5.3-6.4) } \\
\text { with Diabetes High Risk (IDRS } \geq 6 \text { ) }\end{array}$} & \multicolumn{3}{|c|}{$\begin{array}{l}\text { Normoglycemia (A1c <5.3) } \\
\text { with Diabetes High Risk (IDRS } \geq 60 \text { ) }\end{array}$} \\
\hline & & Pre, mg/dL & Post, mg/dL & Diff (\%) & Pre, mg/dL & Post, mg/dL & Diff $(\%)$ & Pre, mg/dL & Post, mg/dL & Diff (\%) \\
\hline \multirow{11}{*}{ Yoga } & TC $(<150)$ & $131.35 \pm 15.76$ & $167.62 \pm 37.67$ & $-36.27 *+(27.6 \%)$ & $130.25 \pm 16.20$ & $160.94 \pm 37.02$ & $-30.69 *(23.5 \%)$ & $131.80 \pm 15.30$ & $163.83 \pm 39.55$ & $\begin{array}{l}-32.03 * \\
(24.3 \%)\end{array}$ \\
\hline & TC (150-200) & $175.32 \pm 14.18$ & $176.61 \pm 38.97$ & $-1.29(0.73 \%)$ & $174.25 \pm 13.93$ & $174.92 \pm 36.88$ & $-0.67+(0.38 \%)$ & $175.61 \pm 13.72$ & $174.50 \pm 35.34$ & $1.11(0.63 \%)$ \\
\hline & $\mathrm{TC}(>200)$ & $228.71 \pm 24.20$ & $185.83 \pm 37.61$ & $42.87 *+(18.7 \%)$ & $230.24 \pm 25.64$ & $187.10 \pm 39.79$ & $43.14 *+(18.7 \%)$ & $232.64 \pm 27.93$ & $187.55 \pm 39.07$ & $45.09 *(19.3 \%)$ \\
\hline & TG $(<150)$ & $104.97 \pm 26.33$ & $147.60 \pm 71.72$ & $-42.62 *+(40.6 \%)$ & $105.63 \pm 25.70$ & $143.61 \pm 67.49$ & $-37.98 *+(35.9 \%)$ & $104.96 \pm 25.49$ & $148.03 \pm 70.88$ & $\begin{array}{l}-43.07^{*} \\
(41.0 \%)\end{array}$ \\
\hline & TG (150-200) & $173.51 \pm 14.58$ & $155.25 \pm 71.17$ & $18.26^{*}+(10.5 \%)$ & $171.58 \pm 14.19$ & $155.81 \pm 61.34$ & $15.77 *+(9.19 \%)$ & $173.06 \pm 14.55$ & $169.29 \pm 81.49$ & $3.77(2.17 \%)$ \\
\hline & TG $(>200)$ & $266.78 \pm 56.77$ & $164.65 \pm 74.78$ & $102.18 *+(38.3 \%)$ & $267.97 \pm 60.35$ & $173.76 \pm 81.09$ & $94.21 *+(35.1 \%)$ & $262.82 \pm 55.22$ & $168.69 \pm 80.60$ & $94.13 *(35.8 \%)$ \\
\hline & LDL $(<100)$ & $76.68 \pm 15.96$ & $95.04 \pm 34.41$ & $-18.36 *+(23.9 \%)$ & $76.55 \pm 16.76$ & $92.66 \pm 33.15$ & $-16.11 *(21.0 \%)$ & $76.56 \pm 17.02$ & $92.19 \pm 31.17$ & $\begin{array}{l}-15.37^{*} \\
(20.0 \%)\end{array}$ \\
\hline & LDL (100-130) & $113.79 \pm 8.53$ & $101.32 \pm 31.96$ & $12.47 *+(10.9 \%)$ & $113.06 \pm 8.18$ & $98.34 \pm 31.88$ & $14.72 *+(13.0 \%)$ & $113.91 \pm 8.27$ & $100.67 \pm 31.93$ & $13.23 *(11.6 \%)$ \\
\hline & LDL (>130) & $152.75 \pm 20.71$ & $107.06 \pm 35.31$ & $45.68 *+(29.9 \%)$ & $154.14 \pm 21.15$ & $108.14 \pm 35.04$ & $46.0 *+(29.8 \%)$ & $155.41 \pm 24.62$ & $108.25 \pm 35.03$ & $47.15 *(30.3 \%)$ \\
\hline & HDL $(<45)$ & $38.69 \pm 5.26$ & $47.01 \pm 11.43$ & $-8.31 *+(21.4 \%)$ & $38.48 \pm 5.17$ & $45.98 \pm 11.34$ & $-7.5(19.4 \%)$ & $38.70 \pm 5.24$ & $46.34 \pm 11.32$ & $\begin{array}{l}-7.64 * \\
(19.7 \%)\end{array}$ \\
\hline & HDL $(>60)$ & $67.86 \pm 6.74$ & $51.36 \pm 11.51$ & $16.49 *+(24.3 \%)$ & $67.66 \pm 6.94$ & $51.25 \pm 11.56$ & $16.41 *+(24.2 \%)$ & $69.07 \pm 9.11$ & $50.84 \pm 12.40$ & $18.23 *(26.3 \%)$ \\
\hline
\end{tabular}


Table 3. Cont.

\begin{tabular}{|c|c|c|c|c|c|c|c|c|c|c|}
\hline \multirow[t]{2}{*}{ Groups } & \multirow{2}{*}{$\begin{array}{l}\text { Lipid } \\
\text { Categories }\end{array}$} & \multicolumn{3}{|c|}{ Diabetes A1c ( $\geq 6.5)$} & \multicolumn{3}{|c|}{$\begin{array}{c}\text { Prediabetes (A1c 5.3-6.4) } \\
\text { with Diabetes High Risk (IDRS } \geq 60 \text { ) }\end{array}$} & \multicolumn{3}{|c|}{$\begin{array}{c}\text { Normoglycemia (A1c <5.3) } \\
\text { with Diabetes High Risk (IDRS } \geq 60 \text { ) }\end{array}$} \\
\hline & & Pre, $\mathrm{mg} / \mathrm{dL}$ & Post, mg/dL & Diff (\%) & Pre, mg/dL & Post, mg/dL & Diff $(\%)$ & Pre, mg/dL & Post, mg/dL & Diff (\%) \\
\hline \multirow{11}{*}{ Control } & $\mathrm{TC}(<150)$ & $130.65 \pm 15.57$ & $170.51 \pm 40.59$ & $-39.86 *(30.5 \%)$ & $130.40 \pm 14.89$ & $167.51 \pm 37.52$ & $-37.10 *(28.4 \%)$ & $128.57 \pm 14.05$ & $180.68 \pm 52.30$ & $\begin{array}{l}-52.11 * \\
(40.5 \%)\end{array}$ \\
\hline & TC (150-200) & $175.53 \pm 14.17$ & $182.58 \pm 41.71$ & $-7.05 *(4 \%)$ & $175.82 \pm 13.77$ & $182.46 \pm 40.62$ & $-6.63 *(3.8 \%)$ & $173.26 \pm 14.12$ & $181.01 \pm 42.40$ & $\begin{array}{l}-7.74 * \\
(4.46 \%)\end{array}$ \\
\hline & TC $(>200)$ & $228.75 \pm 27.34$ & $224.04 \pm 44.16$ & $4.70 *(2.0 \%)$ & $227.27 \pm 24.48$ & $221.15 \pm 40.59$ & $6.11 *(2.7 \%)$ & $229.43 \pm 25.02$ & $225.69 \pm 40.98$ & $3.74 *(1.63 \%)$ \\
\hline & TG $(<150)$ & $104.76 \pm 25.95$ & $\begin{array}{c}162.69 \pm \\
102.67\end{array}$ & $-57.93 *(55.2 \%)$ & $106.41 \pm 26.33$ & $\begin{array}{c}174.32 \pm \\
111.57\end{array}$ & $-67.90 *(63.8 \%)$ & $106.68 \pm 27.13$ & $\begin{array}{c}164.42 \pm \\
100.67\end{array}$ & $\begin{array}{l}-57.73^{*} \\
(54.1 \%)\end{array}$ \\
\hline & TG (150-200) & $173.09 \pm 14.65$ & $158.38 \pm 80.19$ & $14.71 *(3.18 \%)$ & $173.60 \pm 15.59$ & $167.25 \pm 86.19$ & $6.34 *(3.6 \%)$ & $177.69 \pm 16.44$ & $165.00 \pm 88.74$ & $12.69(7.1 \%)$ \\
\hline & TG $(>200)$ & $278.24 \pm 64.15$ & $287.10 \pm 75.54$ & $-8.85 *(3.1 \%)$ & $274.04 \pm 64.22$ & $284.63 \pm 74.75$ & $-10.59 *(3.8 \%)$ & $278.12 \pm 67.55$ & $288.13 \pm 75.20$ & $\begin{array}{l}-10.01 * \\
(4.5 \%)\end{array}$ \\
\hline & LDL $(<100)$ & $76.79 \pm 16.08$ & $95.78 \pm 33.91$ & $-18.98 *(24.7 \%)$ & $76.71 \pm 16.03$ & $100.65 \pm 36.26$ & $-23.94 *(31.2 \%)$ & $75.95 \pm 16.96$ & $98.89 \pm 34.74$ & $\begin{array}{l}-22.94 * \\
(30.2 \%)\end{array}$ \\
\hline & LDL (100-129) & $114.44 \pm 8.47$ & $99.44 \pm 34.25$ & $14.99 *(13.0 \%)$ & $113.98 \pm 8.61$ & $100.90 \pm 38.79$ & $13.08 *(11.4 \%)$ & $114.78 \pm 8.68$ & $94.11 \pm 34.02$ & $20.67 *(18.0 \%)$ \\
\hline & LDL (>130) & $151.63 \pm 18.93$ & $146.32 \pm 37.31$ & $5.31 *(3.5 \%)$ & $149.74 \pm 16.28$ & $143.78 \pm 18 ; 25$ & $5.96 *(3.98 \%)$ & $151.70 \pm 18.68$ & $145.87 \pm 39.92$ & $5.82(3.8 \%)$ \\
\hline & HDL $(<45)$ & $38.86 \pm 5.00$ & $38.21 \pm 7.53$ & $0.44(1.1 \%)$ & $38.13 \pm 4.98$ & $37.03 \pm 7.13$ & $1.10 *(2.8 \%)$ & $37.90 \pm 4.64$ & $38.25 \pm 6.61$ & $-0.34(0.89 \%)$ \\
\hline & HDL (>60) & $67.90 \pm 6.08$ & $51.45 \pm 11.88$ & $16.44 *(24.2 \%)$ & $68.24 \pm 6.21$ & $49.75 \pm 11.85$ & $18.48 *(27.0 \%)$ & $67.28 \pm 5.00$ & $50.92 \pm 11.66$ & $16.35 *(24.3 \%)$ \\
\hline
\end{tabular}

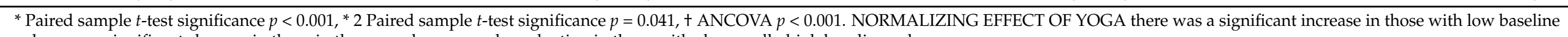
values; non-significant changes in those in the normal range; and a reduction in those with abnormally high baseline values. 
Table 4. Changes in lipid profile in two groups after three months in gender, area (rural/urban), and age subgroups.

\begin{tabular}{|c|c|c|c|c|c|c|c|c|c|c|c|c|c|c|}
\hline \multicolumn{2}{|c|}{ Groups } & \multirow[b]{2}{*}{$\mathrm{Y} / \mathrm{C}$} & \multicolumn{3}{|c|}{ TC (mg/dL) } & \multicolumn{3}{|c|}{ TG (mg/dL) } & \multicolumn{3}{|c|}{ LDL (mg/dL) } & \multicolumn{3}{|c|}{ HDL (mg/dL) } \\
\hline & & & Pre & Post & Df & Pre & Post & Df & pre & Post & Df & pre & Post & $\mathrm{df}$ \\
\hline \multirow{4}{*}{ Gender } & \multirow{2}{*}{ Male } & $\mathrm{Y}$ & $181.96 \pm 39.77$ & $177.44 \pm 38.43$ & 4.52 & $150.02 \pm 69.88$ & $154.33 \pm 73.82$ & -4.31 & $103.5 \pm 34.72$ & $98.73 \pm 33.36$ & 4.78 & $49.3 \pm 11.53$ & $48.90 \pm 11.83$ & 0.48 \\
\hline & & $\mathrm{C}$ & $181.24 \pm 39.63$ & $192.73 \pm 47.73$ & -11.49 & $153.74 \pm 77.62$ & $189.80 \pm 108.57$ & -36.06 & $104.88 \pm 32.90$ & $109.07 \pm 41.25$ & -4.19 & $49.30 \pm 11.55$ & $44.77 \pm 12.26$ & 4.52 \\
\hline & \multirow{2}{*}{ Female } & $\mathrm{Y}$ & $181.68 \pm 39.74$ & $176.03 \pm 38.70 *$ & 5.64 & $150.73 \pm 71.01$ & $152.89 \pm 72.17$ & -2.16 & $103.56 \pm 33.61$ & $98.59 \pm 33.90 *$ & 4.96 & $49.24 \pm 11.44$ & $48.39 \pm 11.34$ & 0.84 \\
\hline & & $\mathrm{C}$ & $184.96 \pm 40.75$ & $193.65 \pm 46.95$ & -8.68 & $157.34 \pm 80.60$ & $192.04 \pm 106.65$ & -34.69 & $103.37 \pm 33.05$ & $107.28 \pm 39.88$ & -3.90 & $48.66 \pm 11.51$ & $44.51 \pm 12.08$ & 4.15 \\
\hline \multirow{3}{*}{ Location } & \multirow{2}{*}{ Urban } & $\mathrm{Y}$ & $181.30 \pm 39.74$ & $176.66 \pm 38.74$ & 4.63 & $150.16 \pm 7070.62$ & $153.87 \pm 73.05$ & -3.70 & $103.07 \pm 34.11$ & $98.54 \pm 33.84$ * & 4.53 & $49.41 \pm 11.39$ & $48.66 \pm 11.64$ & 0.75 \\
\hline & & $\mathrm{C}$ & $183.30 \pm 40.00$ & $193.99 \pm 47.13^{*}$ & -10.69 & $154.43 \pm 79.19$ & $188.01 \pm 105.64$ & -33.58 & $104.35 \pm 33.18$ & $107.7 \pm 40.14$ * & -3.41 & $49.00 \pm 11.67$ & $44.94 \pm 12.40$ * & 4.06 \\
\hline & Rural & $\mathrm{C}$ & $183.59 \pm 40.70$ & $192.48 \pm 47.42$ & -8.8 & $157.45 \pm 79.63 *$ & $194.56 \pm 109.32$ & -37.11 & $103.5 \pm 32.79 *$ & $108.2 \pm 40.80 *$ & -4.69 & $48.83 \pm 11.37$ & $44.26 \pm 11.87^{*}$ & 4.57 \\
\hline \multirow{4}{*}{$\begin{array}{l}\text { Age } \\
\text { groups }\end{array}$} & $<40$ & $\mathrm{Y}$ & $180.80 \pm 38.78$ & $177.0 \pm 38.38 *$ & 3.78 & $149.63 \pm 73.17$ & $150.10 \pm 70.10$ & -0.46 & $102.28 \pm 33.28$ & $99.62 \pm 33.40$ & 2.65 & $49.33 \pm 11.16$ & $48.83 \pm 11.21$ & 0.50 \\
\hline & $<40$ & $\mathrm{C}$ & $182.42 \pm 40.51$ & $195.43 \pm 46.12$ & -11.00 & $155.05 \pm 78.79$ & $191.03 \pm 109.61$ & -35.98 & $104.28 \pm 32.65$ & $107.91 \pm 40.93$ & -3.63 & $49.30 \pm 11.52$ & $44.88 \pm 12.46$ & 4.42 \\
\hline & $>40$ & $\mathrm{Y}$ & $182.18 \pm 39.93$ & $176.49 \pm 38.85^{*}$ & 5.68 & $150.71 \pm 69.49$ & $154.90 \pm 73.79 *$ & -4.18 & $103.92 \pm 34.33$ & $98.28 \pm 33.92 *$ & 5.64 & $49.34 \pm 11.54$ & $48.55 \pm 11.54^{*}$ & 0.79 \\
\hline & $>40$ & $\mathrm{C}$ & $183.10 \pm 40.27$ & $192.53 \pm 47.65^{*}$ & -9.43 & $156.14 \pm 79.63$ & $191.15 \pm 106.70$ * & -35.00 & $103.89 \pm 33.12$ & $108.0 \pm 40.29 *$ & -4.15 & $48.79 \pm 11.53$ & $44.53 \pm 12.05^{*}$ & 4.26 \\
\hline
\end{tabular}

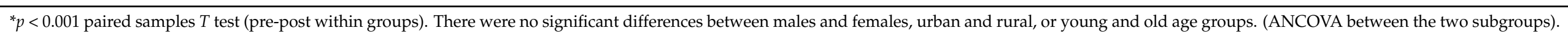




\section{Discussion}

\subsection{Yoga as an Effective Tool}

After three months of intervention, there was a noteworthy decline in the blood lipid (TC, TG, LDL) of subjects with diabetes, prediabetes, and non-diabetes. Interestingly, the values in all three groups showed a similar trend with a significant increase in those with low levels and a decrease in high values in the yoga group, e.g., there was a significant increase in those with HDL $<45 \mathrm{mg} / \mathrm{dL}$, while the levels decreased in those with high values of $\geq 60 \mathrm{mg} / \mathrm{dL}$. A similar regulating effect was seen in TC, TG, and LDL, pointing to the regulatory effect of yoga in normalizing the values to reach a healthy status, i.e., increasing if it was low and decreasing if it was high, which has not been reported earlier.

Similar effects were observed in the case of both males and females. After three months of intervention, a reduction in the vital lipid parameters such as TC and LDL was observed for both the subgroups (male and female). However, TG was found to be elevated in both genders. Rural and urban populations differ in some of the basic characteristics such as relative pollution, lifestyle, diet, and stress. It was observed that yoga induced the same level of changes in biochemical markers in urban populations as in rural populations. Age is one of the important contributing risk factors associated with diabetes [32]. We categorized the study population into two groups based on age, i.e., below or above 40 years. Analysis on the basis of this categorization revealed that yoga is as effective in the aged population ( $>40 \mathrm{yrs})$ as in the younger population ( $<40 \mathrm{yrs})$.

Several studies have shown the positive effects of yoga in T2DM, although none had used a national consensus protocol [33]. A study on the effect of pranayama and yogasanas on blood glucose and lipid profile in a two-armed design on 60 patients of T2DM between 35-60 yrs with diabetes recruited from the diabetes clinic of a hospital in Delhi, India, had shown a significant reduction in serum insulin, blood glucose (fasting and postprandial), LDL, TG, and VLDL with an increase in HDL, with insignificant changes in the control group after 45 days [34]. A short-term study based on asanas, relaxation, and pranayama had also shown a reduction in the lipid profile within nine days of intervention [35]. A systematic review of original studies on the metabolic and clinical effects of yoga in adults with T2DM summarized the beneficial effects of several variables, including anthropometric, blood pressure, glucose tolerance, insulin sensitivity, etc. This study concluded that to realign the global focus towards yoga, better quality studies using standardized yoga programs are required to validate the effects in populations with T2DM [24].

The lipid regulating effect of the integrated yoga lifestyle module has been highlighted in this study as noted by the significantly higher number of subjects shifting from high or low values to the normal range. The state of health, as cited in yoga literature, is defined as a state of dynamically changing functioning of the tissues to achieve a balance (samatvam yoga) under varying internal (psychological or biochemical) or external (environmental) situations [36]. Yoga masters evolved several techniques to achieve mastery over the mind (chittavrittinirodhaha) that monitors lifestyle behavior through mindfulness [37]. Healing during disease is through restoring this (samatvam) harmony or homeostasis. Yoga offers several techniques for correcting the imbalance at mental, emotional, and physical levels, which are postulated to manifest in biochemical changes [38]. Several studies on yoga have shown a similar harmonizing effect. For example, an improved autonomic balance was seen in healthy volunteers [39] and those with heightened sympathetic tone [40]. Studies have also shown restoration of diurnal cortisol rhythm in patients [41].

The efficacy of yoga in achieving this metabolic biochemical homeostasis through lipid normalizing effect opens up several research questions about the mechanism of yoga. Future studies are imperative at the cellular level to examine whether yoga improves LDL receptor sensitivity or receptor-mediated endocytosis and receptor recycling based on the feedback regulation of receptors [42]. Whether the regulatory effect of yoga on HDL is mediated through a reverse cholesterol transport mechanism [43] that includes macrophage cholesterol efflux in arteries or an antioxidant or anti-inflammatory effect [44-46] mediated by NO promoting activity needs to be evaluated [43]. The increased number of diabetes 
cases in the Indian population creates a substantial economic burden [47-50] and poses a threat to having age-related disorders [51-53]. Therefore, the study may be extrapolated to other populations based on the varied acceptability of yoga protocol as its perceived benefits, barriers, and compliance may vary from one country to another. However, the generalizability of the yoga protocol to the other parts of the country may also depend on the availability and acceptability of certified yoga practitioners. Besides, it is difficult to predict the sustainability of the intervention for which long-term studies are required, although the benefits experienced by the participants are likely to attract them in long-term practice. With the establishment of 150,000 wellness centers in the country [54], the longterm sustainability and generalizability to other populations of the intervention may also depend on the extent to which it is integrated with modern medicine and prescribed by physicians or public health enthusiasts.

\subsection{Strengths}

This is the first multicenter nationwide study in both rural and urban populations on the effect of yoga lifestyle on lipid levels. Additionally, this intervention involved certified yoga therapists (checked by inter-rater reliability testing) from several member institutions of the IYA. There were no adverse/serious events reported during the intervention period of three months. However, a few incidents of minor events like pain in the knee or spine or generalized body pain, or issues related to digestion were reported. These issues were handled by the yoga instructors by correcting their postures and advising on relaxation or counter postures.

\subsection{Limitations of the Study}

As this was an interventional study, double-blinding was not possible as the instructor and the participant did know that they were doing yoga as a therapy. The post values of lipid profile were not available in all those who participated due to various reasons: (a) dropouts from the study after recruitment because of time constraints; (b) left the residence in the recruited cluster zone for business or change of jobs although they had participated in $80 \%$ of the sessions; (c) blood samples were corrupted (hemolyzed) in a few cases; (d) post data could not be collected due to local conditions such as weather (heavy snowfall (Kashmir valley), extremes of temperature $\left(50{ }^{\circ} \mathrm{C}\right.$ in Odisha), heavy rainfall (western ghats of Karnataka) etc.).

Although efforts were made to ensure reliability by several levels of supervision to promote punctuality and uniformity of the intervention, we could not ensure perfect monitoring due to weather conditions (Odisha and Kashmir), local political disturbances (election drive in UP), state-level agitation (Manipur), transfer of yoga volunteers (Kerala and Goa), etc.

\section{Conclusions}

The data presented in this study prompts more detailed investigations, including molecular genetics and cell culture approaches, to understand the mechanism of yoga protocols on lipid metabolism in general. The results indicate a non-redundant impact of yoga intervention, calling for its integration in medical institutes, further increasing the scope of convenience sampling through larger interdisciplinary studies on patients with dyslipidemia and diabetes. Further, with the launch of 150,000 wellness centers by the Government of India, these trained instructors could be used to provide daily modules of DYP, assisted by ASHA and multipurpose workers. Their services could also be utilized for screening and control of other non-communicable diseases. In this manner, regular yoga sessions in each district could lead to health promotion, prevention, and management of diabetes. As these centers will represent a triage of modern medicine, AYUSH, and science investigators, such integration could further lead to increased acceptance and incorporation into the NPCDCS in an evidence-based manner through new protocols for cancer palliative care, cardiovascular disease, and stroke prevention. 
Supplementary Materials: The following are available online at https:/ / www.mdpi.com/article/10.339 0/medicines8070037/s1, Figure S1: Map of India showing the geographic spread of the intervention sites (reusing the figure after permission (Nagendra et al., 2019), Figure S2: Graphs showing the normalizing effect of yoga, Table S1: Validated yoga lifestyle protocol for prediabetes and uncomplicated diabetes.

Author Contributions: R.N.: proposal writing, planning, monitoring, conducting, review, analysis and editing; S.K.: original writing; A.A.: concept of manuscript; I.N.A.: concept, review; A.K.S.: planning, monitoring, data management and quality assurance; S.S.P.: data segregation and analysis; R.H.L.: data collection, monitoring, quality assurance; P.D.: data collection, monitoring, quality assurance; H.R.N.: vision, concept, proposal, planning, monitoring, advice, problem solving, editing. All authors have read and agreed to the published version of the manuscript.

Funding: We thank Ministry of Health and Family and Ministry of AYUSH (through CCRYN), Govt. of India for funding this project. The funding was received via grant letter number F.No. 16-63/2016-17/CCRYN/RES/Y\&D/MCT/dated 15.12.2016.

Ethical Compliance: Screening and recruitment were carried out after getting the permission from IEC, reference no RES/IEC-IYA/001 dated 16th Dec 2016. Written informed consent was taken before the assessment. The study was primarily done by S-VYASA under IYA.

Institutional Review Board Statement: The study was conducted according to the guidelines of the Declaration of Helsinki, and ap-proved by the Ethics Committee of Indian Yoga Association (protocol code RES/IEC-IYA/001 and date of approval 16-12-2016).

Informed Consent Statement: Informed consent was obtained from all subjects involved in the study.

Data Availability Statement: All the associated data is available within the manuscript/Supplementary Materials.

Acknowledgments: We are thankful to (a) the funding by the Ministry of health and family welfare and the Ministry of AYUSH routed through Central Council for Research in Yoga and Naturopathy, Govt, of India for their timely support of this project (b) the executive committee of Indian yoga Association for conducting NMB (c) Art of Living Institute, Vethathiri Maharishi College of Yoga, Patanjali Yogpeeth, PGI Chandigarh, and SVYASA for providing more than 1200 volunteers and (d) the members of the research advisory board of NMB for their inputs at all stages of the study.

Conflicts of Interest: The authors declare no conflict of interest.

\author{
Abbreviations \\ DYP Diabetes yoga lifestyle protocol \\ NMB Niyantrita Madhumeha Bharata Abhiyaan \\ NABL National Accreditation Board for Testing and Calibration Laboratories \\ A1c Glycated hemoglobin \\ TC Total cholesterol \\ TG Triglyceride \\ LDL Low-density lipoprotein \\ HDL High-density lipoprotein \\ VLDL Very-low-density lipoprotein \\ T2DM Type-2 diabetes mellitus \\ CHD Coronary heart disease \\ ADA American Diabetes Association \\ ANS Autonomic nervous system \\ SAM Sympatho-adrenal medullary \\ HPA Hypothalamic pituitary adrenal \\ NO Nitric oxide \\ IDRS Indian diabetes risk score \\ IYA Indian Yoga Association \\ CEB Census enumeration blocks \\ SRF Senior research fellows \\ ASHA Accredited social health activists \\ IEC Institutional ethics committee
}




\section{References}

1. Mithal, A.; Majhi, D.; Shunmugavelu, M.; Talwarkar, P.G.; Vasnawala, H.; Raza, A.S. Prevalence of dyslipidemia in adult Indian diabetic patients: A cross sectional study (SOLID). Indian J. Endocrinol. Metab. 2014, 18, 642-647. [CrossRef]

2. Rosenson, R.S.; Brewer, H.B.; Ansell, B.; Barter, P.; Chapman, M.J.; Heinecke, J.W.; Kontush, A.; Tall, A.R.; Webb, N.R. Translation of high-density lipoprotein function into clinical practice: Current prospects and future challenges. Circulation 2013, 128, 1256-1267. [CrossRef] [PubMed]

3. Frank, A.T.; Zhao, B.; Jose, P.O.; Azar, K.M.; Fortmann, S.P.; Palaniappan, L.P. Racial/Ethnic Differences in Dyslipidemia Patterns. Circulation 2014, 129, 570-579. [CrossRef] [PubMed]

4. $\quad \mathrm{Pu}$, J.; Romanelli, R.; Zhao, B.; Azar, K.M.; Hastings, K.G.; Nimbal, V.; Fortmann, S.P.; Palaniappan, L.P. Dyslipidemia in Special Ethnic Populations. Cardiol. Clin. 2015, 33, 325-333. [CrossRef]

5. Chandra, K.S.; Bansal, M.; Nair, T.; Iyengar, S.; Gupta, R.; Manchanda, S.C.; Mohanan, P.; Rao, V.D.; Manjunath, C.; Sawhney, J.; et al. Consensus statement on management of dyslipidemia in Indian subjects. Indian Heart J. 2014, 66, S1-S51. [CrossRef] [PubMed]

6. Farmer, J.A. Diabetic dyslipidemia and atherosclerosis: Evidence from clinical trials. Curr. Diabetes Rep. 2008, 8, 71-77. [CrossRef] [PubMed]

7. Association, A.D. Management of dyslipidemia in adults with diabetes. Diabetes care 2003, 26, s83-s86.

8. Wilson, P.W.; D'Agostino, R.B.; Parise, H.; Sullivan, L.; Meigs, J.B. Metabolic Syndrome as a Precursor of Cardiovascular Disease and Type 2 Diabetes Mellitus. Circulation 2005, 112, 3066-3072. [CrossRef]

9. Joshi, S.R.; Anjana, R.M.; Deepa, M.; Pradeepa, R.; Bhansali, A.; Dhandania, V.K.; Joshi, P.P.; Unnikrishnan, R.; Nirmal, E.; Subashini, R.; et al. Prevalence of Dyslipidemia in Urban and Rural India: The ICMR-INDIAB Study. PLoS ONE 2014, 9, e96808. [CrossRef]

10. Fox, C.S.; Coady, S.; Sorlie, P.D.; D’Agostino, R.B.; Pencina, M.J.; Vasan, R.S.; Meigs, J.B.; Levy, D.; Savage, P.J. Increasing Cardiovascular Disease Burden Due to Diabetes Mellitus. Circulation 2007, 115, 1544-1550. [CrossRef]

11. Silverio, A.; Cavallo, P.; De Rosa, R.; Galasso, G. Big Health Data and Cardiovascular Diseases: A Challenge for Research, an Opportunity for Clinical Care. Front. Med. 2019, 6, 36. [CrossRef]

12. Saha, S.; Mondal, S.; Kundu, B. Yoga as a therapeutic intervention for the management of type 2 diabetes mellitus. Int. J. Yoga 2018, 11, 129-138. [CrossRef] [PubMed]

13. Tundwala, V.; Gupta, R.P.; Kumar, S.; Singh, V.B.; Sandeep, B.R.; Dayal, P.; Prakash, P. A study on effect of yoga and various asanas on obesity, hypertension and dyslipidemia. Int. J. Basic Appl. Med. Sci. 2012, 2, 93-98.

14. Chandrasekaran, A.M.; Kinra, S.; Ajay, V.S.; Chattopadhyay, K.; Singh, K.; Singh, K.; Praveen, P.A.; Soni, D.; Devarajan, R.; Kondal, D.; et al. Effectiveness and cost-effectiveness of a Yoga-based Cardiac Rehabilitation (Yoga-CaRe) program following acute myocardial infarction: Study rationale and design of a multi-center randomized controlled trial. Int. J. Cardiol. 2019, 280, 14-18. [CrossRef] [PubMed]

15. Kumar, S.; Sudha, S.; Chopra, M.; Khan, F.; Sharma, K. Physical Exercise and Yoga: As an alternative approach towards COVID-19 management. Curr. Tradit. Med. 2021, 7, 1. [CrossRef]

16. Yeung, A.C.; Chang, D.H.T.; Bensoussan, A.; Kiat, H. Yoga and cardiac rehabilitation: A brief review of evidence. J. Yoga Phys. Ther. 2015, 5, 1000207. [CrossRef]

17. Bali, P.; Kaur, N.; Tiwari, A.; Bammidi, S.; Podder, V.; Devi, C.; Kumar, S.; Sivapuram, M.S.; Ghani, A.; Modgil, S.; et al. Effectiveness of Yoga as the Public Health Intervention Module in the Management of Diabetes and Diabetes Associated Dementia in South East Asia: A Narrative Review. Neuroepidemiology 2020, 54, 287-303. [CrossRef]

18. Pal, D.K.; Bhalla, A.; Bammidi, S.; Telles, S.; Kohli, A.; Kumar, S.; Devi, P.; Kaur, N.; Sharma, K.; Kumar, R.; et al. Can Yoga-Based Diabetes Management Studies Facilitate Integrative Medicine in India Current Status and Future Directions. Integr. Med. Int. 2017, 4, 125-141. [CrossRef]

19. Arati, M.; Arpita, P.; Arati, M. Effect of yoga (asana and pranayama) on serum lipid profile in normal healthy volunteers. Int. J. Contemp. Med. Res. 2015, 2, 1277-1281.

20. Behar, S.; Graff, E.; Reicher-Reiss, H.; Boyko, V.; Benderly, M.; Shotan, A.; Brunner, D. Low total cholesterol is associated with high total mortality in patients with coronary heart disease. Eur. Heart J. 1997, 18, 52-59. [CrossRef]

21. Elmehdawi, R.R. Hypolipidemia: A word of caution. Libyan J. Med. 2008, 3, 84-90. [CrossRef] [PubMed]

22. Shantakumari, N.; Sequeira, S.; El Deeb, R. Effects of a yoga intervention on lipid profiles of diabetes patients with dyslipidemia. Indian Heart J. 2013, 65, 127-131. [CrossRef] [PubMed]

23. Damodaran, A.; Malathi, A.; Patil, N.; Shah, N.; Marathe, S. Therapeutic potential of yoga practices in modifying cardiovascular risk profile in middle aged men and women. J. Assoc. Physicians India 2002, 50, 633-640.

24. Innes, K.E.; Selfe, T.K. Yoga for Adults with Type 2 Diabetes: A Systematic Review of Controlled Trials. J. Diabetes Res. 2016, 2016, 1-23. [CrossRef]

25. Raveendran, A.V.; Deshpandae, A.; Joshi, S.R. Therapeutic Role of Yoga in Type 2 Diabetes. Endocrinol. Metab. 2018, 33, 307-317. [CrossRef] [PubMed]

26. Gotto Jr, A.M. Cholesterol management in theory and practice. Circulation 1997, 96, 4424-4430. [CrossRef]

27. Mahesh, N.K.; Kumar, A.; Bhat, K.G.; Verma, N. Role of yoga therapy on lipid profile in patients of hypertension and prehypertension. Int. J. Adv. Med. 2018, 5, 321. [CrossRef] 
28. Mohammed, R.; Banu, A.; Irman, S.; Jaiswal, R.K. Importance of yoga in diabetes and dyslipidemia. Int. J. Res. Med. Sci. 2016, 4, 3504-3508. [CrossRef]

29. Nagendra, H.R.; Nagarathna, R.; Rajesh, S.K.; Amit, S.; Telles, S.; Hankey, A. Niyantrita Madhumeha Bharata 2017, methodology for a nationwide diabetes prevalence estimate: Part 1. Int. J. Yoga 2019, 12, 179-192. [CrossRef]

30. Weber, M.B.; Ranjani, H.; Staimez, L.R.; Anjana, R.M.; Ali, M.; Narayan, K.V.; Mohan, V. The Stepwise Approach to Diabetes Prevention: Results From the D-CLIP Randomized Controlled Trial. Diabetes Care 2016, 39, 1760-1767. [CrossRef]

31. Nagarathna, R.; Rajesh, S.K.; Amit, S.; Patil, S.; Anand, A.; Nagendra, H.R. Methodology of Niyantrita Madhumeha Bharata Abhiyaan- 2017, a nationwide multicentric trial on the effect of a validated culturally acceptable lifestyle intervention for primary prevention of diabetes: Part 2. Int. J. Yoga 2019, 12, 193-205. [CrossRef]

32. Sattar, N.; Rawshani, A.; Franzén, S.; Rawshani, A.; Svensson, A.M.; Rosengren, A.; McGuire, D.K.; Eliasson, B.; Gudbjörnsdottir, S. Age at diagnosis of type 2 diabetes mellitus and associations with cardiovascular and mortality risks: Findings from the Swedish National Diabetes Registry. Circulation 2019, 139, 2228-2237. [CrossRef]

33. Kaur, N.; Malik, N.; Mathur, D.; Pal, S.; Malik, R.; Rana, S. Mindfulness and yoga halt the conversion of pre-diabetic rural women into diabetics-a pilot study. Integr. Med. Case Rep. 2020, 1, 8-18. [CrossRef]

34. Singh, S.; Kyizom, T.; Singh, K.P.; Tandon, O.P.; Madhu, S.V. Influence of pranayamas and yoga-asanas on serum insulin, blood glucose and lipid profile in type 2 diabetes. Indian J. Clin. Biochem. 2008, 23, 365-368. [CrossRef] [PubMed]

35. Bijlani, R.L.; Vempati, R.P.; Yadav, R.K.; Ray, R.B.; Gupta, V.; Sharma, R.; Mehta, N.; Mahapatra, S.C. A Brief but Comprehensive Lifestyle Education Program Based on Yoga Reduces Risk Factors for Cardiovascular Disease and Diabetes Mellitus. J. Altern. Complement. Med. 2005, 11, 267-274. [CrossRef]

36. Satyapriya, M.; Nagarathna, R.; Padmalatha, V.; Nagendra, H. Effect of integrated yoga on anxiety, depression \& well being in normal pregnancy. Complement. Ther. Clin. Pr. 2013, 19, 230-236. [CrossRef]

37. Satyapriya, M.; Nagendra, H.R.; Nagarathna, R.; Padmalatha, V. Effect of integrated yoga on stress and heart rate variability in pregnant women. Int. J. Gynecol. Obstet. 2008, 104, 218-222. [CrossRef] [PubMed]

38. Madanmohan, A.B.B.; Sanjay, Z.; Dayanidy, G.; Basavaraddi, I.V. Effect of yoga therapy on reaction time, biochemical parameters and wellness score of peri and post-menopausal diabetic patients. Int. J. Yoga 2012, 5, 5-10. [CrossRef]

39. Udupa, K.; Sathyaprabha, T.N.; Telles, S.; Singh, N. Influence of Yoga on the Autonomic Nervous System. In Research-Based Perspectives on the Psychophysiology of Yoga; IGI Global: Hershey, PA, USA, 2018; pp. 67-85.

40. Sathyaprabha, T.; Kisan, R.; Adoor, M.; Nalini, A.; Kutty, B.M.; Murthy, B.C.; Sujan, M.; Rao, R.; Raju, T. Effect of Yoga on migraine: A comprehensive study using clinical profile and cardiac autonomic functions. Int. J. Yoga 2014, 7, 126-132. [CrossRef]

41. Rao, R.M.; Vadiraja, H.; Nagaratna, R.; Gopinath, K.S.; Patil, S.; Diwakar, R.B.; Shahsidhara, H.; Ajaikumar, B.; Nagendra, H. Effect of yoga on sleep quality and neuroendocrine immune response in metastatic breast cancer patients. Indian J. Palliat. Care 2017, 23, 253-260. [CrossRef]

42. Goldstein, J.L.; Brown, M.S. History of Discovery: The LDL receptor. Arterioscler. Thromb. Vasc. Biol. 2009, 29, 431-438. [CrossRef]

43. Rosenson, R.S.; Brewer, H.B.; Davidson, W.S.; Fayad, Z.A.; Fuster, V.; Goldstein, J.; Hellerstein, M.; Jiang, X.C.; Phillips, M.C.; Rader, D.J.; et al. Cholesterol efflux and atheroprotection: Advancing the concept of reverse cholesterol transport. Circulation 2012, 125, 1905-1919. [CrossRef]

44. Pal, R.; Gupta, N. Yogic practices on oxidative stress and of antioxidant level: A systematic review of randomized controlled trials. J. Complement. Integr. Med. 2017, 16. [CrossRef] [PubMed]

45. Manna, I. Effects of yoga training on body composition and oxidant-antioxidant status among healthy male. Int. J. Yoga 2018, 11, 105-110. [CrossRef] [PubMed]

46. Shete, S.U.; Verma, A.; Kulkarni, D.D.; Bhogal, R.S. Effect of yoga training on inflammatory cytokines and C-reactive protein in employees of small-scale industries. J. Educ. Health Promot. 2017, 6, 76. [CrossRef] [PubMed]

47. Javalkar, S.R. The economic burden of health expenditure on diabetes mellitus among urban poor: A cross sectional study. Int. J. Community Med. Public Health 2019, 6, 1162-1166. [CrossRef]

48. Podder, V.; Srivastava, V.; Kumar, S.; Nagarathna, R.; Sivapuram, M.S.; Kaur, N.; Sharma, K.; Singh, A.K.; Malik, N.; Anand, A.; et al. Prevalence and Awareness of Stroke and Other Comorbidities Associated with Diabetes in Northwest India. J. Neurosci. Rural. Pr. 2020, 11, 467-473. [CrossRef]

49. Kumar, S.; Anand, A.; Nagarathna, R.; Kaur, N.; Sivapuram, M.S.; Pannu, V.; Pal, D.K.; Malik, N.; Singh, A.K.; Nagendra, H.R. Prevalence of prediabetes, and diabetes in Chandigarh and Panchkula region based on glycated haemoglobin and Indian diabetes risk score. Endocrinol. Diabetes Metab. 2021, 4, 162. [CrossRef]

50. Goyal, A.K.; Bhadada, S.; Malik, N.; Anand, A.; Kumar, R.; Bammidi, S.; Tyagi, R.; Modgil, S.; Sharma, K.; Bali, P.; et al. Guinness world record attempt as a method to pivot the role of Yoga in Diabetes management. Ann. Neurosci. 2019, 26, 21-24. [CrossRef]

51. Anand, A.; Banik, A.; Thakur, K.; Masters, C.L. The Animal Models of Dementia and Alzheimer's Disease for Pre-Clinical Testing and Clinical Translation. Curr. Alzheimer Res. 2012, 9, 1010-1029. [CrossRef]

52. Sharma, N.K.; Gupta, A.; Prabhakar, S.; Singh, R.; Sharma, S.K.; Chen, W.; Anand, A. Association between CFH Y402H Polymorphism and Age Related Macular Degeneration in North Indian Cohort. PLoS ONE 2013, 8, e70193. [CrossRef] [PubMed] 
53. Nagarathna, R.; Tyagi, R.; Kaur, G.; Vendan, V.; Acharya, I.N.; Anand, A.; Singh, A.; Nagendra, H.R. Efficacy of a Validated Yoga Protocol on Dyslipidemia in Diabetes Patients: NMB-2017 India Trial. Medicines 2019, 6, 100. [CrossRef] [PubMed]

54. Ved, R.R.; Gupta, G.; Singh, S. India's health and wellness centres: Realizing universal health coverage through comprehensive primary health care. WHO South-East Asia J. Public Health 2019, 8, 18-20. [CrossRef] [PubMed] 